\title{
Evaluation of Flexible Graphical User Interface for Intuitive Human Robot Interactions
}

\author{
Balázs Dániel $^{1}$, Péter Korondi ${ }^{1}$, Gábor Sziebig ${ }^{2}$, Trygve \\ Thomessen $^{3}$
}

${ }^{1}$ Department of Mechatronics, Optics, and Mechanical Engineering Informatics, Budapest University of Technology and Economics, PO BOX 91, 1521 Budapest, Hungary

e-mail: daniel@mogi.bme.hu; korondi@mogi.bme.hu

2 Department of Industrial Engineering, Narvik University College, Lodve Langes gate 2, 8514 Narvik, Norway

e-mail: gabor.sziebig@hin.no

${ }^{3}$ PPM AS, Leirfossveien 27, 7038 Trondheim, Norway

e-mail: trygve.thomessen@ppm.no

\begin{abstract}
A new approach for industrial robot user interfaces is necessary due to the fact that small and medium sized enterprises are more interested in automation. The increasing number of robot applications in small volume production requires new techniques to ease the use of these sophisticated systems. In this paper shop floor operation is in the focus. A Flexible Graphical User Interface is presented which is based on cognitive infocommunication (CogInfoCom) and implements the Service Oriented Robot Operation concept. The definition of CogInfoCom icons is extended by the introduction of identification, interaction and feedback roles. The user interface is evaluated with experiments. Results show that a significant reduction in task execution time and a lower number of required interactions is achieved because of the intuitiveness of the system with human centered design.
\end{abstract}

Keywords: industrial robotics; human robot interaction; cognitive infocommunication; flexible robot cell; graphical user interface; usability

\section{Introduction}

In the era of touch screen based smartphones the interaction between humans and machines is becoming a frequent event. Especially the communication between people and robots is a field of research nowadays. Human Robot Interaction (HRI) studies are aiming the user friendly application of different robot systems for 
cooperation with humans. This includes an interactive conversation robot which collects multi-modal data and bonds with human partners. [1]

While the interaction with an industrial robot is traditionally considered as a Human Machine Interaction (HMI) due to its inferior level of autonomy and complexity [2], these systems are also spreading to human environments. Shared workspace [3], teach by demonstration [4], all these improvements in industrial robotics require a higher level of communication. The need for better and more intuitive user interfaces for industrial manipulators is increasing.

The reason of the increasing interest is that a wide range of Small and Medium Sized Enterprises (SMEs) are motivated to invest in industrial robot systems and automation due to increasing cost levels in western countries. The last years a significant part of robot installations in industry required high flexibility and high accuracy for low-volume production of SMEs [5]. This tendency adds new challenges to support and transfer knowledge between robotics professionals and SMEs. The difficulty of operating a robotic manipulator is present both on shopfloor production and remote support for the robot cell. Operators with less expertise will supervise the robot cells in SMEs because of economical reasons and there is usually no dedicated robot system maintenance group for small companies. Remote operation of the industrial robot systems from the system integrator's office offers an alternative solution. This is based on the premise that if one can perform remote assistance for the SMEs, the enterprise can get high reliability, high productivity, faster and cheaper support irrespective of geographical distance between the enterprise and the system integrator or other supportive services. On the other hand the need for assistance from professionals can be reduced by introducing such user/operator interfaces which fit better for inexperienced users and provide an intuitive and flexible manner of operation.

The paper is organized as follows: Section 2 briefly summarizes the properties of Human Robot Interaction for industrial robots. Section 3 presents a Flexible Graphical User Interface implementation test with quantitative and qualitative results.

\section{Human Robot Interaction}

Scholtz [2] proposed five different roles for humans in HRI. Supervisor, operator, mechanic, teammate and bystander; these define the necessary level of information exchange from both sides. In order to keep the interaction continuous the switch between roles is inevitable in certain situations. Efficient user interfaces have to take into account the adequate display of details in complex systems like robot cells. 
Automated systems may be designed in machine-centered or human-centered way [6]. While the first technique requires high level knowledge from the user for operation, the latter is more adaptable for flexible systems which serves both seasoned and unseasoned personnel. Standard HRI for industrial equipment is usually utilize machine-centered approach whereas complex systems are accessible through complicated user interfaces.

Human-centered design applies multidisciplinary knowledge, thus combining the necessary factors and benefits both for machinery and human operation. The ISO Standard 9241-210:2010 [7] defines it as an activity to increase productivity in parallel with improved work conditions by the use of ergonomics and human factors. User friendly or human friendly systems are designed with the aim of minimizing the machine factor. This implies that in this case robots have to adapt to the operator, but the difficulty is to provide a comprehensive and appropriate human behavior model [8].

Human factors include psychological aspects also. HRI should be analyzed from this point of view to make a better match between robot technology and humans. The systematic study of the operator's needs and preferences is inevitable [9] to meet the expectations on psychological level, moreover, in order to satisfy these needs, multimodal user interfaces are necessary which may facilitate the communication with intuitive and cognitive functions $[9,10]$.
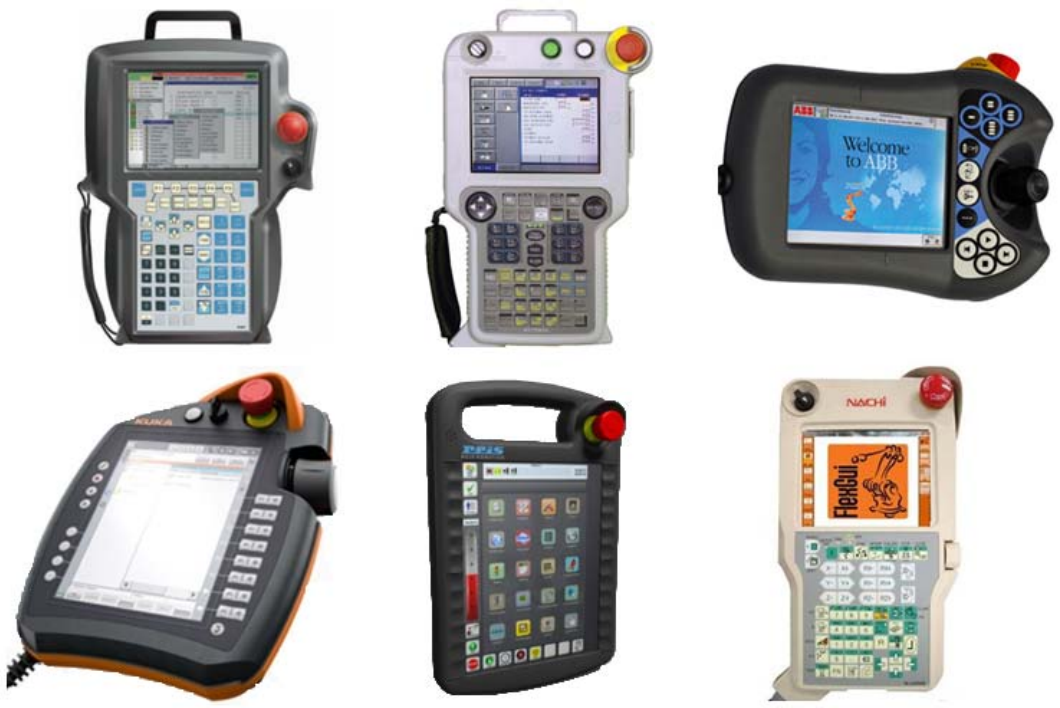

Figure 1

Modern Teach Pendants.

From top-left corner: FANUC iPendant [11], Yaskawa Motoman NX100 teach pendant [12], ABB FlexPendant [13], KUKA smartPad [14], ReisPAD [15], Nachi FD11 teach pendant 
For industrial robotic manipulators the standard user interface is the Teach Pendant. It is a mobile, hand-held device usually with customized keyboard and graphical display. Most robot manufacturers are developing distinctive design (See Figure 1) and are including a great number of features thus increasing the complexity and flexibility.

Input methods vary from key-centric to solely touch screen based. Feedback and system information is presented on graphical display in all cases, however the operator may utilize a great number of other information channels; touch, vision, hearing, smell and taste. The operator can also take an advantage of the brain's ability to integrate the information acquired from his or her senses. Thus, although the operator has the main sight and attention oriented towards the robot's tool, she will immediately change her attention to any of the robot's link colliding with an obstacle when it is intercepted by her peripheral vision.

The science of integrating informatics, info-communication and cognitivesciences, called CogInfoCom is investigating the advantages of combining information modalities and has working examples in virtual environments, robotics and telemanipulation $[10,16]$. The precise definition and description of CogInfoCom is presented in [17], terminology is provided in [18], while [19] walks the reader through the history and evolution of CogInfoCom.

Introducing this concept in the design of industrial robot user interfaces is a powerful tool to meet the goal of human-centred systems and a more efficient human robot interaction.

\section{Flexible Graphical User Interface}

At first the focus is laid on the possible improvements regarding the existing Teach Pendant information display. The traditional industrial graphical user interfaces are designed to deal with a large number of features, thus the organization of menus and the use of the system is complex and complicated.

In a real life example the robot cell operator had to ask assistance from the system integrator in a palletizing application because the manipulator constantly moved to wrong positions. Without clear indication on the robot's display the user could not recognize that the programmed positions are shifted due to the fact that the system is in the middle of the palletizing sequence. The resetting option was in two menulevels deep in the robot controller's constant settings. In case of a flexible user interface this could have been avoided by providing custom surface for palletizing instead of showing the robot program, the date, etc.

In most cases the customization of the robot user interface is possible. A separate computer and software is required [20] and due to the complexity of the system it 
is done by the system integrator. Naturally, the integrator cannot be fully aware of the needs of the operator thus the flexibility of a robot cell is depending also on the capabilities of the framework in which the graphical user interface is working. The communication barrier between the operator and the integrator influences the efficiency of the production through inadequate and non-optimized information flow (See Figure 2). Differences in competence level, geographical distance or cultural distance can all cause difficulties in overcoming this barrier.

The Flexible Graphical User Interface (FGUI) concept aims to close this gap. The system integrator still have the opportunity to compile task specific user interfaces but the framework includes pre-defined, robot specific elements which are at the operators' hands at all times. The functionality is programmed by the integrator and represented as she thinks it is the most fitting, but the final information channel can be rearranged by the user/operator to achieve efficient and human (operator) centered surface.

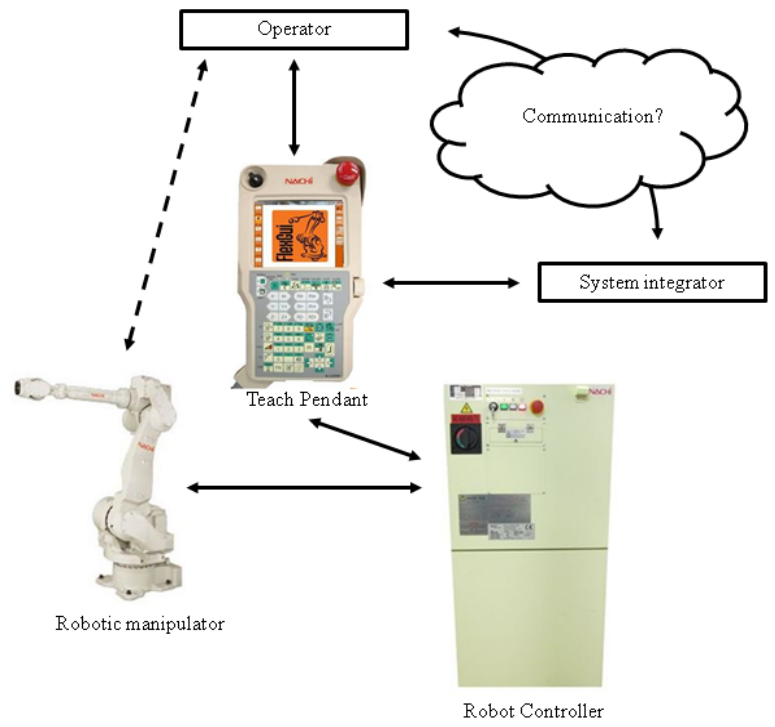

Figure 2

Connection of a robot system with the operator and the integrator

\subsection{Shop-floor operation}

During shop-floor operation the user should communicate on high level with the robot cell. In high volume and highly automated production lines this interaction is restricted the best to start a program at the beginning of the worker's shift and stop it when the shift is over. For SMEs this approach is not feasible: frequent reconfiguration and constant supervision is inevitable. Therefore a service- 
oriented user interface is favourable which hides the technical details of a multipurpose robot cell behind an abstract level. This abstraction should be made by utilizing the principles of CogInfoCom and allowing intuitive use of a robot cell.

The connections between the actual robot programs and the operator's user interface are presented in Figure 3. As the system keeps track of the parts in a pick and place operation this data is stored as a set of variables. The operator can observe these values by traditional display items but a FGUI allows to present it in a different way: small lamps are giving visual information of the system's state. The operator instinctively and instantly can compare the actual state of the robot cell with the information stored in the robot controller and detects defects faster. This is an application of representation-bridging in CogInfoCom, because the information on the robot variable is still visual but the representation is different from standard robot variable monitoring. The direct observation of variables would require the knowledge of the system integrator's convention of data representation from the operator.

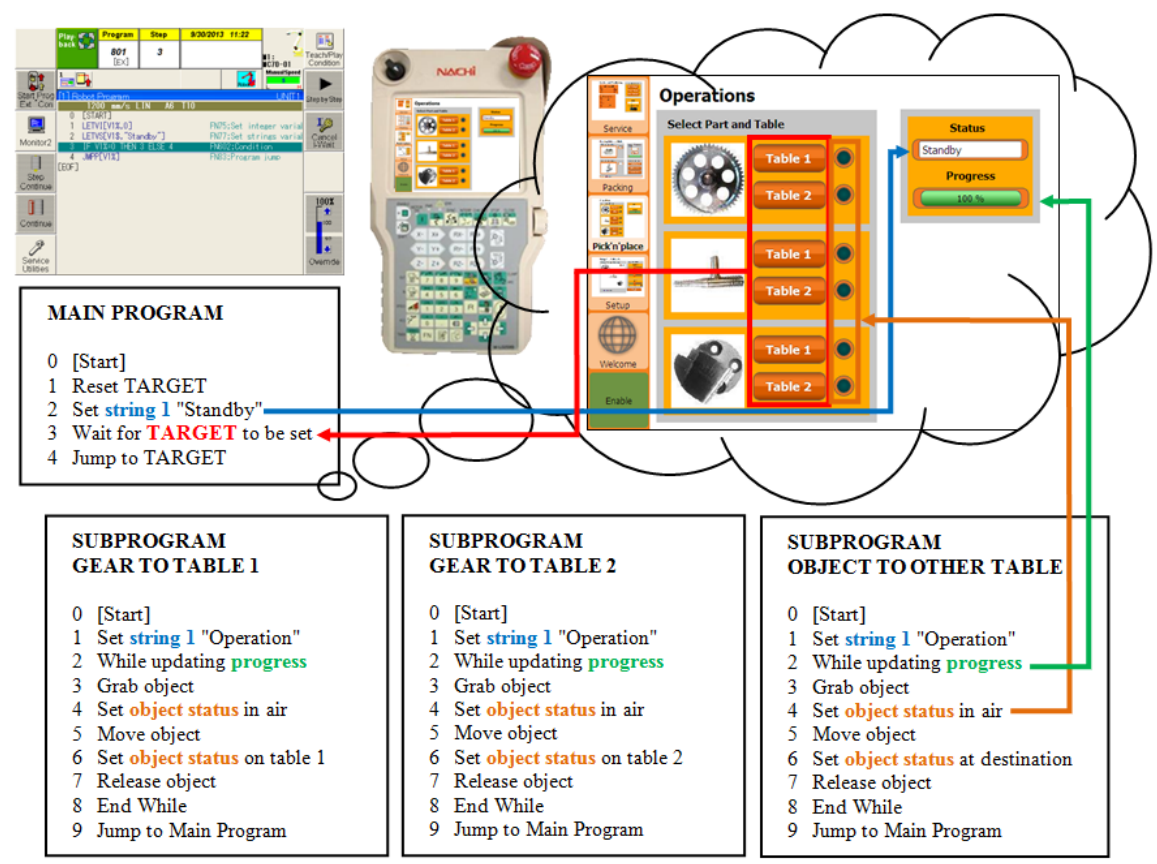

Figure 3

Connections between functionality and service-oriented layers

The selection of programs for the operation is reduced to service requests initiated by button presses paired with an image of the part. Using CogInfoCom semantics [18]; the images of parts, the buttons, the indicator lamps, the progress 
bar and the text box are all visual icons bridging the technical robot data with operation parameters and statuses. The high-level message generated by these entities is the list of achievable item movements in case of a pick and place service and feedback on the current operation provided by the user interface not from visual observation of the cell thus we consider this application of CogInfoCom channel as an eyecon message transferring system in general.

Furthermore, considering a group of screen elements (e.g. the image of the gear, the buttons "Table 1" and "Table 2" and the indicator lamps) we use low-level direct conceptual mapping. The picture of the part generates an instant identification of the robot service objective and the surface to interact with the robot controller. The buttons represent identification and also interaction with the robot cell. The robot system internal mapping of real world is sent back to the user through the indicator lamps in feedback form.

These applications indicates that CogInfoCom icons usually not only generate and represent a message in the communication but these also have roles. These roles may depend on the actual implementation and concept transmitted by messages. In human robot interaction three main roles may be distinguished:

- identification role,

- interaction role,

- feedback role.

The simple instruction "Move the gear from Table 1 to Table 2" given to the operator does not require additional mapping from the user thus the human robot interaction is simplified and became human centered. The gear may be identified by image, manipulated by interaction with the button and get feedback though the indicator lamp.

Practically the user interface implements an abstract level between the technical realization of operations and the service oriented operation. A main robot program is monitoring the inputs from the user. By pressing one of the buttons a number is loaded into a variable which causes to controller to change to the program indicated by this number. The program contains the pre-defined pose values to be executed sequentially. The current program line number divided by the total number of lines indicates the progress with task for the user in the progress bar, since one program contains only one item movement from one table to another. Exiting the main program also sets the text box to "Operation in progress" and reentering it resets to "Standby".

The robot controller keeps track of objects in the robot cell by adjusting an integer variable according to the current position of it in the motion sequence. Values 1, 2 and 0 represent the item placed on Table 1, placed on Table 2, and lifted and moved by the robot, respectively. Mapping this information onto the indicator lamps means that when the object is on one of the tables, the lamp next to the 
table button lights up, sending the message that this table is occupied. When it is in the air both lamps are dimmed.

It is generally important to rate the success of a concept and its implementations with experiments. The assessment in communication improvement is may be measured both quantitatively and qualitatively.

\subsection{Concept evaluation with user tests}

Justification of the service-based flexible user interface is performed by a series of testing with real users. It was a comparative test of a traditional and very conservative system and a newly developed flexible graphical user interface. The robot cell was set up for two different services which are the following:

- $\quad$ pick and place operation with two positions on three work pieces,

- parameterizable delivery system for bolting parts.

\subsubsection{Test description}

Test participants executed two main tasks first using the traditional system (Figure 4) then repeating them using the flexible user interface (Figure 5 and Figure 6). During the tests user interactions with the robot system were recorded by cameras, key logging, and screen capturing.

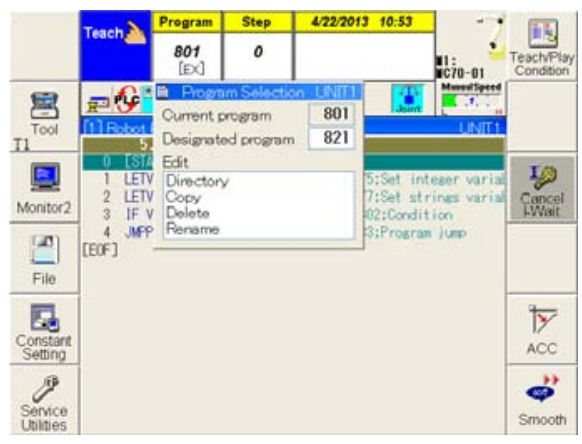

Figure 4

Traditional Graphical User Interface

At the beginning of each task a briefing was given to the participants on the general aim of the executed task (Table 1). After that they read through a summary on the necessary settings (Table 2 and Table 3 ) to be made before the robot can perform its operation. At this point participants had the opportunity to go on with a user manual with step-by-step instruction on the necessary steps or continue on their own. Chosen the second option they always had the chance to refer to the manual if they needed suggestions on how to continue. 


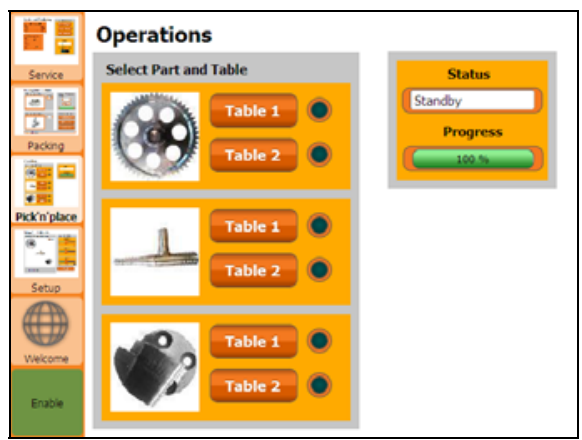

Figure 5

Flexible Graphical User Interface for Task 3

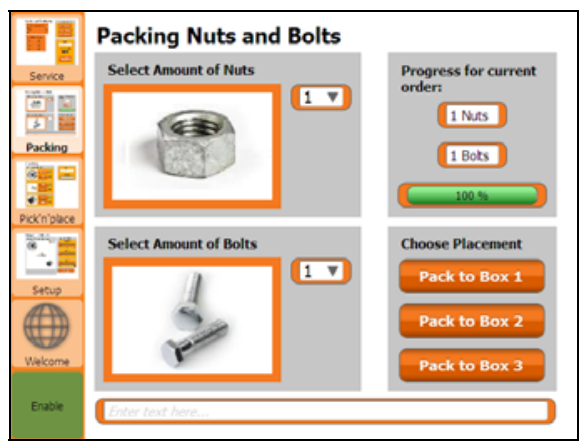

Figure 6

Flexible Graphical User Interface for Task 4

During the execution of Task 1 the only action needed was to select the appropriate program for the robot based upon the required part movement. Task 2 was more complicated; three internal variables had to be set according to the number of nuts and bolts and the number of the delivery box.

The participant was provided with the new user interface for the execution of Task 3 and Task 4 thus the direct interaction with robot controller variables and setting were obscured. 
Table 1

General aims of tasks

\begin{tabular}{|l|l|c|}
\hline Task ID & Description & Interface $^{1}$ \\
\hline $\begin{array}{l}\text { Task 1.1 } \\
\text { Task } 1.2\end{array}$ & $\begin{array}{l}\text { Move the pipe from Table } 1 \text { to Table } 2 \\
\text { Move the cutting head from Table } 2 \text { to Table } 1\end{array}$ & TGUI \\
\hline Task 2 & Deliver two bolts and three nuts to Box1 & TGUI \\
\hline $\begin{array}{l}\text { Task } 3.1 \\
\text { Task } 3.2\end{array}$ & $\begin{array}{l}\text { Move the pipe from Table } 2 \text { to Table } 1 \\
\text { Move the cutting head from Table } 1 \text { to Table } 2\end{array}$ & FGUI \\
\hline Task 4 & Deliver two bolts and three nuts to Box 1 & FGUI \\
\hline
\end{tabular}

Table 2

Settings for Task 1

\begin{tabular}{|l|c|c|c|}
\hline Item & Source & Destination & Program Number \\
\hline \multirow{2}{*}{ Pipe } & Table 1 & Table 2 & 816 \\
\cline { 2 - 4 } & Table 2 & Table 1 & 815 \\
\hline \multirow{2}{*}{ Cutting head } & Table 1 & Table 2 & 814 \\
\cline { 2 - 4 } & Table 2 & Table 1 & 813 \\
\hline \multirow{2}{*}{ Gear } & Table 1 & Table 2 & 812 \\
\cline { 2 - 4 } & Table 2 & Table 1 & 811 \\
\hline
\end{tabular}

Table 3

Settings for Task 2

\begin{tabular}{|l|c|c|}
\hline Parameter & Variable & Value \\
\hline Program & Program Number & 821 \\
\hline Number of nuts & Integer Nr. 014 & 3 \\
\hline Number of bolts & Integer Nr. 015 & 2 \\
\hline Delivery box ID & Integer Nr. 016 & 1 \\
\hline
\end{tabular}

\subsubsection{Results}

The evaluation was conducted with four participants, all male, between age 25 and 27. All four have engineering background; two have moderate, two have advanced experience in robot programming. Participants were advised that audio and video is recorded which serves only for scientific analysis and they were assured that the test can be interrupted anytime on their initiation.

All participants were able to execute all of the tasks in a reasonable amount of time. Two users reported difficulties to set the program number during Task 1.2. The problem turned out to be a software bug in the traditional robot controller user interface; the user manual had been modified accordingly, although none of the

\footnotetext{
${ }^{1}$ TGUI: Traditional Graphical User Interface, FGUI: Flexible Graphical User Interface
} 
participants followed the user manual steps strictly, most likely due to their previous experience with robots.

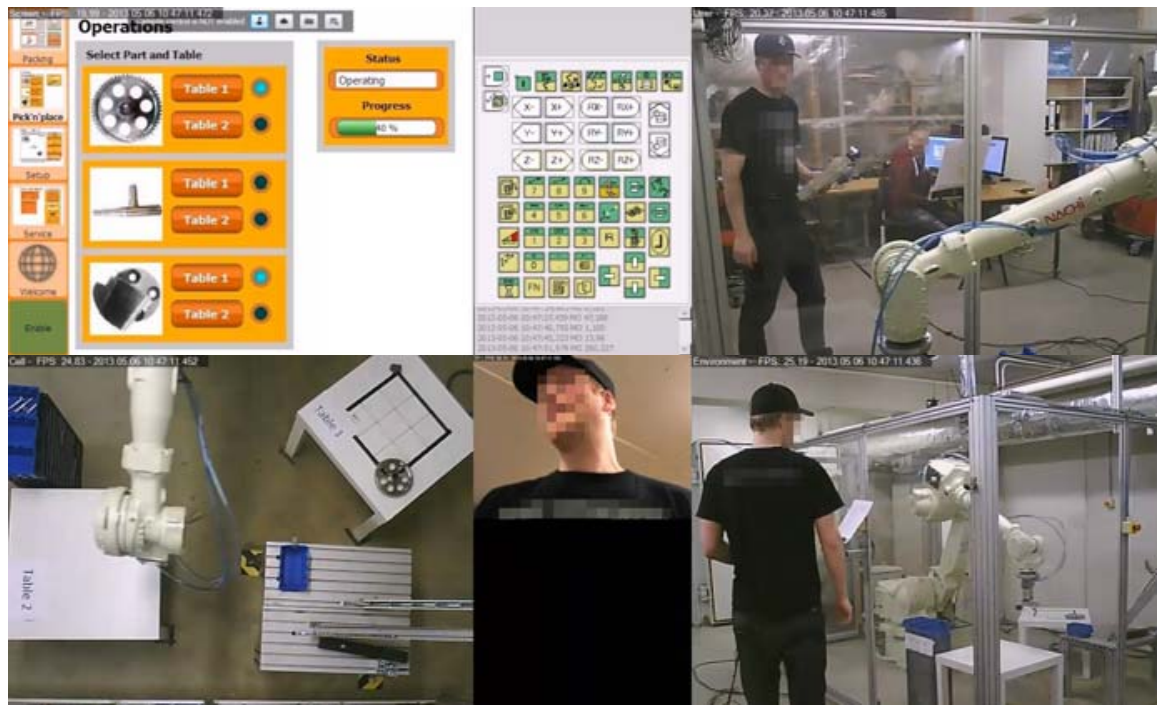

Figure 7

Synchronised videos had been taken during testing

Collected data have been evaluated after the tests. Three parameters have been selected to represent the difference between the traditional and the flexible approach: task execution duration, number of interactions and ratio of number of key presses to touch screen commands.

Execution time is measured between the first interaction with the Teach Pendant recorded by the key logger and mouse logger running on the robot controller, and the last command which ordered the robot to move. The last interaction was determined by time stamp on the video in case of the TGUI measurement, since logging of program start button on the controller housing was not in place at the time of the experiment. The start of robot movement could be determined from mouse logging data in case of FGUI.

The mean execution time using the conservative system for Task 1.1, Task 1.2 and Task 2 are 36, 37 and 135 seconds respectively. In contrast, Task 3.1, Task 3.2 and Task 4 duration was 31, 0 and 30 seconds with the use of the FGUI. Data are presented in Table 4 . The total mean time spent on Task 1 and Task 3 are 72,1 and 38,1 second respectively. All the mean values presented based on three measurements. Due to the low number of measurements further statistical data were not computed. 
Table 4

Task execution time

\begin{tabular}{|l|c|c|c|r|r|c|}
\cline { 2 - 7 } \multicolumn{1}{c|}{} & \multicolumn{7}{c|}{ Task duration [s] } \\
\cline { 2 - 7 } \multicolumn{1}{c|}{} & 1.1 & 1.2 & 2 & \multicolumn{1}{c|}{3.1} & \multicolumn{1}{c|}{$3.2^{2}$} & \multicolumn{1}{c|}{4} \\
\hline User 1 & 50,0 & 53,5 & 131,5 & 64,5 & 0 & 21,7 \\
\hline User 2 & 25,2 & 30,9 & 121,0 & 27,9 & 0 & 11,1 \\
\hline User 3 & 31,6 & 25,1 & 151,3 & 21,8 & 0 & 22,4 \\
\hline Mean & $\mathbf{3 5 , 6}$ & $\mathbf{3 6 , 5}$ & $\mathbf{1 3 4 , 6}$ & $\mathbf{3 8 , 1}$ & $\mathbf{0}$ & $\mathbf{1 8 , 4}$ \\
\hline User 4 $^{3}$ & N/A & N/A & 126,3 & 9,4 & 0 & 63,8 \\
\hline
\end{tabular}

Participants had to configure the robot controller using the Teach Pendant which offered two possible interactions: key presses and touch screen interactions. A virtual representation of the keyboard is depicted in Figure 7. Besides, users had to deactivate the joint brakes and energize the motors as well as start the robot program by pressing buttons on the robot controller housing. All interactions were counted by the logging software and later the touch interaction ratio to key presses was calculated as follows:

$T R[\%]=\frac{n_{\text {touch }}}{n_{\text {touch }}+n_{\text {keypress }}+n_{\text {controller }}} \cdot 100$,

where $n_{\text {touch }}$ is the number of touch screen interactions, $n_{\text {keypress }}$ is the number of button presses on the Teach Pendant and $n_{\text {controller }}$ is the number of button presses on the controller housing. The total number of interactions and touch ratio is listed in Table 5. The data loss in execution time measurement did not affect counting of interactions thus mean values are calculated from four samples.

Table 5

Number of interactions and touch ratio

\begin{tabular}{|l|l|c|c|c|c|r|}
\cline { 2 - 7 } \multicolumn{1}{c|}{} & \multicolumn{6}{c|}{ Interactions [-] (Touch ratio [\%]) } \\
\cline { 2 - 7 } \multicolumn{1}{c|}{} & 1.1 & 1.2 & 2 & 3.1 & \multicolumn{1}{c|}{3.2} & \multicolumn{1}{c|}{4} \\
\hline User 1 & $7(14)$ & $21(19)$ & $57(11)$ & $7(71)$ & $1(100)$ & $10(100)$ \\
\hline User 2 & $7(14)$ & $10(30)$ & $33(15)$ & $6(50)$ & $1(100)$ & $6(100)$ \\
\hline User 3 & $7(14)$ & $14(50)$ & $53(15)$ & $6(83)$ & $1(100)$ & $8(100)$ \\
\hline User 4 & $7(14)$ & $13(23)$ & $26(17)$ & $3(67)$ & $1(100)$ & $7(71)$ \\
\hline Mean & $\mathbf{7 ( 1 4 )}$ & $\mathbf{1 5 ( 3 1 )}$ & $\mathbf{4 2 ( 1 5 )}$ & $\mathbf{6 ~ ( 6 8 )}$ & $\mathbf{1 ( 1 0 0 )}$ & $\mathbf{8 ( 9 3 )}$ \\
\hline
\end{tabular}

2 Task duration is zero because the first interaction already started the task execution for the robot.

${ }^{3}$ Due to data corruption Task 1.1 and Task 1.2 duration is not available. Mean value is calculated with three measurements in all cases. 


\subsubsection{Discussion}

This testing aimed to evaluate the new concept of service oriented flexible user interface. No particular selection was in place for the participants and the size of dataset is not wide enough for statistical analysis and true usability evaluation [21]. However, trends and impressions can be synthesized on the difference in user performance. Inspection of the video recordings shows the participants did not understand fully that Task 1 and Task 3 as well as Task 2 and Task 4 were the same except the fact that they have to use different user interface during execution. Although in the series of actions they did not follow the step-bystep instruction of the user manual, participants returned to it over and over again to get acknowledgement on their progression. An excessive number of interactions is present against the number of interactions required by the user manual (Table 6).

Table 6

Comparison of required and performed interactions

\begin{tabular}{|l|c|c|c|c|}
\cline { 2 - 5 } \multicolumn{1}{c|}{} & Required & Performed & Difference & Difference [\%] \\
\hline Task 1.1 & 7 & 7 & 0 & $0 \%$ \\
\hline Task 1.2 & 9 & 15 & +6 & $+67 \%$ \\
\hline Task 2 & 25 & 42 & +17 & $+68 \%$ \\
\hline Task 3.1 & 3 & 6 & +3 & $+100 \%$ \\
\hline Task 3.2 & 1 & 1 & 0 & $0 \%$ \\
\hline Task 4 & 6 & 8 & +2 & $+33 \%$ \\
\hline Total & $\mathbf{5 1}$ & $\mathbf{7 9}$ & $+\mathbf{2 8}$ & $\mathbf{+ 5 5 \%}$ \\
\hline
\end{tabular}

At the beginning the state of the user interface and the controller was the same in every test but at the start of Task 1.2 this situation changed due to the different preferences of the users on how to stop a robot program. The significant difference for Task 2 is caused by the fact that there are several ways of inputting a variable in the traditional GUI but the shortest (based upon the robot manufacturer's user manual) was not used by either of the participants.

The excess number of interactions for Task 3 are the actions to dismiss messages on the flexible user interface caused by previous action of the users. The increased number of touches in Task 4 are due to a usability issue: the display item for selecting the amount of parts to be delivered was to small thus the selection could not be made without repeated inputs. Verdict of this investigation is that users tends to use less efficient ways to set up the robot controller which may induce errors and execution time increases due to the need of recuperating from errors. 


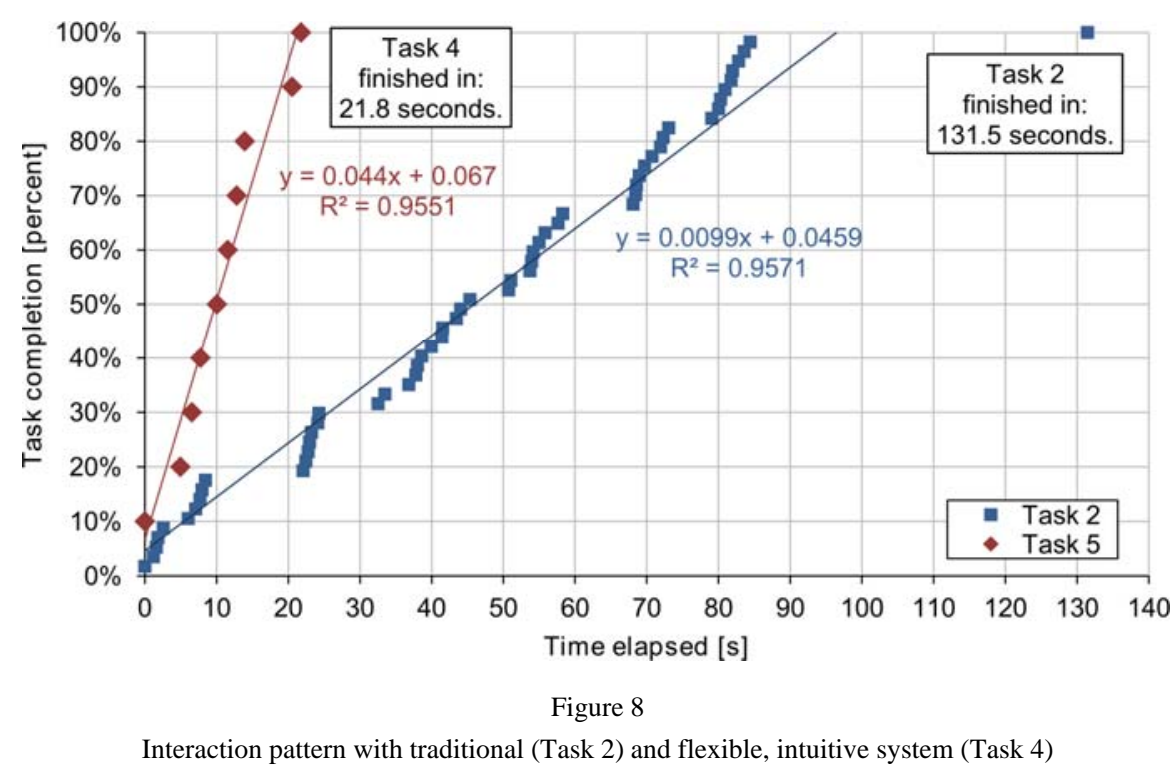

The intuitiveness of the new approach can be proven with the examination of interaction patterns. Figure 8 shows the interactions of User 1 in details. The user input for the traditional system comes in bursts. The slope of each burst is close to the final slope of the FGUI interaction (Task 4 in Figure 8) but the time between these inputs decreases the overall speed of setting. Recordings show that this time in the case of Task 2 is generally spent on figuring out the next step to execute either by looking in the manual or searching for clues on the display. Finally the press of the start button for Task 2 is delayed because the user double-checked the inputted values.

In contrast the inputs for Task 4 are distributed in time uniformly and the delay between the interactions is significantly lower. The user did not have to refer to the user manual because the user interface itself gave enough information for setup. This means that this composition of user interface which is made specifically for this service of the robot cell offers a more intuitive and easy-to-use interface than the traditional one and that CogInfoCom messages were transmitted successfully.

The overall picture shows that FGUI performs significantly better than TGUI (See Figure 9). For comparison the execution time of Task 3 against Task 1 was shortened by 34 seconds while the duration of Task 4 against Task 2 was shorter by 116 seconds. Regarding the necessary interactions with system FGUI reduced it to $23,4 \%$ giving around a quarter of possibilities for errors. 
Total execution time

207

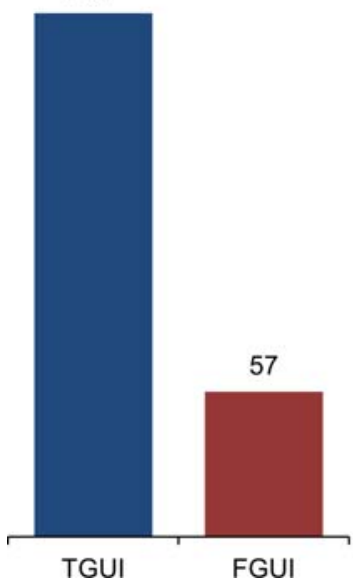

Total number of

interactions

64

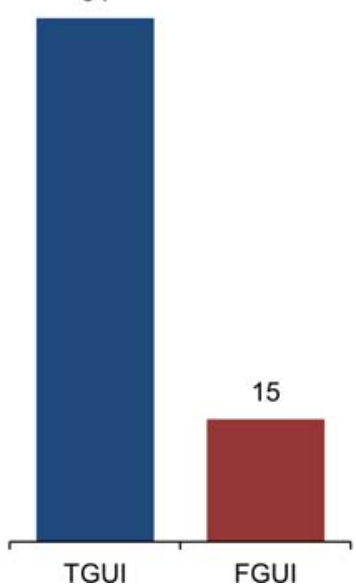

Overall ratio of touch

interactions to key presses

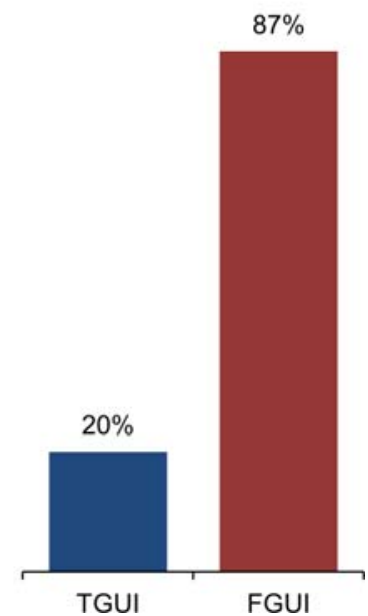

Figure 9

Final results showing improved performance of FGUI: reduced task execution time, decreased number of interaction and increased ratio of touch screen usage to key presses

The Flexible Graphical User Interface also increased the use of touch screen significantly (e.g. from 15\% to 93\% for Task 2 and Task 4). Participants reported that the use of images and the composition of the UI helped them to connect easier the parameters given by the instructions and the necessary actions to input these values into the controller. This is the result of deliberate design of the abstract level connected to the robot cell's service; the design is based upon the principle of CogInfoCom messages to ensure human centred operation.

Concluding the discussion it is stated that this Flexible Graphical User Interface is evaluated as expected; users were able to operate the robot cell faster, more intuitively and with greater self-confidence. Due to the low number of participants further verification is necessary; organisation of a new test for deeper usability and intuitiveness investigation (including non-expert users) is underway at the time of writing this paper and results will be published in later papers.

\section{Conclusions}

The Flexible Graphical User Interface implementation based on Service Oriented Robot Operation was presented. The application of CogInfoCom principles is described and a new property for the CogInfoCom icon notion was introduced. This new property is the role of icon in message transfer and for human robot interaction identification, interaction and feedback roles were identified. 
The new approach represented by Service Oriented Robot Operation in human robot interaction for industrial applications was examined by user evaluation. Results show the decrease in task setup and completion time and the reduced number of interactions proves a more intuitive way of communication between man and machine.

\section{Acknowledgement}

This work was supported by BANOROB project, the project is funded by the Royal Norwegian Ministry of Foreign Affairs. The authors also would like to thank The Norwegian Research Council for supporting this research work through The Industrial PhD program.

\section{References}

[1] J. Scholtz: Theory and evaluation of human robot interactions, System Sciences, 36th Annual Hawaii International Conference on, pp. 10, 2003.

[2] B. Vaughan, J. G. Han, E. Gilmartin, N. Campbell: Designing and Implementing a Platform for Collecting Multi-Modal Data of HumanRobot Interaction, Acta Polytechnica Hungarica, Vol. 9, No. 1, pp. 7-17, 2012.

[3] S. Stadler, A. Weiss, N. Mirnig, M. Tscheligi: Anthropomorphism in the factory - a paradigm change?, Human-Robot Interaction (HRI), 2013 8th ACM/IEEE International Conference on, pp. 231-232, 2013.

[4] B. Solvang, G. Sziebig: On Industrial Robots and Cognitive Infocommunication, Cognitive Infocommunications (CogInfoCom 2012), 3rd IEEE International Conference on, Paper 71, pp. 459-464, 2012.

[5] World Robotics - Industrial Robots 2011, Executive Summary. Online, Available: http://www.roboned.nl/sites/roboned.nl/files/2011_Executive _Summary.pdf, 2011.

[6] G. A. Boy (ed.): Human-centered automation, in The handbook of humanmachine interaction: a human-centered design approach. Ashgate Publishing, pp. 3-8, 2011.

[7] Human-centred design for interactive systems, Ergonomics of humansystem interaction -- Part 210, ISO 9241-210:2010, International Organization for Standardization, Stand., 2010.

[8] Z. Z. Bien, H.-E. Lee: Effective learning system techniques for humanrobot interaction in service environment, Knowledge-Based Systems, Vol. 20, No. 5, pp. 439-456, June 2007.

[9] A. V. Libin, E. V. Libin: Person-robot interactions from the robotpsychologists' point of view: the robotic psychology and robotherapy approach, Proceedings of the IEEE, Vol. 92, No. 11, pp. 1789-1803, Nov. 2004. 
[10] P. Korondi, B. Solvang, P. Baranyi: Cognitive robotics and telemanipulation, 15th International Conference on Electrical Drives and Power Electronics, Dubrovnik, Croatia, pp. 1-8, 2009.

[11] FANUC iPendantTM brochure, FANUC Robotics America Corporation (http://www.fanucrobotics.com), 2013.

[12] Yaskawa Motoman Robotics NX100 brochure, Yaskawa America, Inc. (http://www.yaskawa.com), 2011.

[13] FlexPendant - Die neue Version des Programmierhandgertes bietet noch mehr Bedienkomfort, (in German), ABB Asea Brown Boveri Ltd. (www.abb.com), 2009.

[14] KUKA smartPad website, KUKA Roboter GmbH, (www.kukarobotics.com), 2013.

[15] Smart robot programming, the easy way of programming, Reis GmbH \& Co. KG Maschinenfabrik. (www.reisrobotics.de), 2013.

[16] P. Baranyi, B. Solvang, H. Hashimoto, P. Korondi: 3D Internet for Cognitive Infocommunication, Hungarian Researchers on Computational Intelligence and Informatics, 10th International Symposium of 2009 on, pp. 229-243, 2009.

[17] P. Baranyi, A. Csapo: Definition and Synergies of Cognitive Infocommunications, Acta Polytechnica Hungarica, Vol. 9, No. 1, pp. 6783, 2012.

[18] A. Csapo, P. Baranyi: A Unified Terminology for the Structure and Semantics of CogInfoCom Channels, Acta Polytechnica Hungarica, Vol. 9, No. 1, pp. 85-105, 2012.

[19] G. Sallai: The Cradle of the Cognitive Infocommunications, Acta Polytechnica Hungarica, Vol. 9, No. 1, pp. 171-181, 2012.

[20] B. Daniel, P. Korondi, T. Thomessen: New Approach for Industrial Robot Controller User Interface, Proceedings of the IECON 2013 - 39th Annual Conference of the IEEE Industrial Electronics Society, pp. 7823-7828, 2013.

[21] Wonil Hwang and Gavriel Salvendy: Number of people required for usability evaluation: the $10 \backslash \pm 2$ rule, Communication of the ACM, Vol. 53, No. 5, pp. 130-133, May 2010. 